\title{
Existence of periodic solutions of a $p$-Laplacian-Neumann problem
}

Raad Awad Hameed ${ }^{1,2}$, Jiebao Sun ${ }^{1}$ and Boying $\mathrm{Wu}^{1 *}$

${ }^{\text {*Correspondence: }}$ mathwby@hit.edu.cn 'Department of Mathematics, Harbin Institute of Technology, Harbin, 150001, P.R. China Full list of author information is available at the end of the article

\begin{abstract}
In this paper, we study a periodic $p$-Laplacian equation with nonlocal terms and Neumann boundary conditions. We establish the existence of time periodic solutions of the $p$-Laplacian-Neumann problem by the theory of Leray-Schauder degree.
\end{abstract}

\section{Introduction}

In this paper, we consider the periodic boundary problem for a $p$-Laplacian equation of the following form:

$$
\begin{aligned}
& \frac{\partial u}{\partial t}-\operatorname{div}\left(|\nabla u|^{p-2} \nabla u\right)=(m-\Phi[u]) u, \quad(x, t) \in Q_{T}, \\
& \frac{\partial u}{\partial n}=0, \quad(x, t) \in \partial \Omega \times(0, T), \\
& u(x, 0)=u(x, T), \quad x \in \Omega,
\end{aligned}
$$

where $p>2, \Omega$ is a bounded domain in $\mathbb{R}^{n}$ with smooth boundary $\partial \Omega, \frac{\partial}{\partial n}$ denotes the outward normal derivative on $\partial \Omega, Q_{T}=\Omega \times(0, T)$. This problem is motivated by models which have been proposed for some problems in mathematical biology. The function $u$ represents the spatial densities of the species at $(x, t)$; the diffusion term $\operatorname{div}\left(|\nabla u|^{p-2} \nabla u\right)$ represents the effect of dispersion in the habitat, which models a tendency to avoid crowding, and the speed of the diffusion is slow since $p>2$; the term $(m-\Phi[u]) u$ models the contribution of the population supply due to births and deaths; the Neumann boundary conditions model the trend of the biology population to survive on the boundary. Assumptions of $m, \Phi[u]$ will be introduced in the next section.

The model as (1.1) was first studied by Allegretto and Nistri. In [1] they studied the existence of nontrivial nonnegative periodic solutions and optimal control for the following equation:

$$
\frac{\partial u}{\partial t}-\Delta u=f(x, t, m, \Phi[u], u)
$$

with Dirichlet boundary value conditions. Later, many mathematical researchers studied extended forms of this kind of equation. For example, in [2-7], the authors considered some nonlinear diffusion equations with nonlocal terms such as the porous equation with $m>1$, the $p$-Laplacian equation with $p>2$ and the doubly degenerate parabolic equation.

(c) 2013 Hameed et al.; licensee Springer. This is an Open Access article distributed under the terms of the Creative Commons Attribution License (http://creativecommons.org/licenses/by/2.0), which permits unrestricted use, distribution, and reproduction in any medium, provided the original work is properly cited. 
All these problems are the Dirichlet boundary value conditions, and these boundary conditions describe that the boundary we consider in this model is lethal to the species. Moreover, the methods in these papers are all based on the theory of Leray-Schauder degree. However, there are few results on degenerate periodic parabolic equations with nonlocal terms and Neumann boundary conditions. Recently, in [8], Wang and Yin considered the following periodic Neumann boundary value problem:

$$
\begin{aligned}
& \frac{\partial u}{\partial t}-\Delta u^{m}=(a-\Phi[u]) u, \quad(x, t) \in Q_{T}, \\
& \frac{\partial u}{\partial n}=0, \quad(x, t) \in \partial \Omega \times(0, T), \\
& u(x, 0)=u(x, T), \quad x \in \Omega,
\end{aligned}
$$

where $m>1$. By the parabolic regularized method and the theory of Leray-Schauder degree, they established the existence of nontrivial nonnegative periodic solutions. Also, there are many works about reaction diffusion equations without nonlocal term; one can see [9-13] and the references therein, and the boundary value condition and research method are different from our work.

In this paper, we consider the periodic solution of $p$-Laplacian Neumann problem (1.1)(1.3). In [14], the authors studied equation (1.1) with the Dirichlet boundary value condition. Compared with the Dirichlet boundary value condition in [14], the Neumann boundary value condition causes an additional difficulty in establishing some a priori estimates. On the other hand, different from that in the case of the Dirichlet boundary value condition, the standard regularized problem of problem (1.1)-(1.3) is not well posed, and thus a modified regularized problem for (1.1)-(1.3) is considered. In addition, we will make use of the Moser iterative method to establish the a priori upper bound of the solution of the regularized problem. By the theory of Leray-Schauder degree, we prove that this modified problem admits nontrivial nonnegative periodic solutions. Then, by passing to a limit process, we obtain the existence of nontrivial nonnegative periodic solutions of problem (1.1)-(1.3). In the process of proving the main results, the nonlocal term, which reflects the reality of the model (1.1), will cause a difficulty when we establish a lower bound estimate of the maximum modulus of the solution of the regularized problem. Otherwise, we can use the method of upper and lower solution to prove the existence of periodic solutions. At last, the existence theorem shows that the spatial densities of the species are periodic under the case of nonlinear diffusion.

This paper is organized as follows. In Section 2, we show some necessary preliminaries including the modified regularized problem. In Section 3, we establish some necessary $a$ priori estimations of the solution of the modified regularized problem. Then we obtain the main results of this paper.

\section{Preliminaries}

In this paper, we assume that

(A1) $\Phi[\cdot]: L_{+}^{2}(\Omega) \rightarrow \mathbb{R}^{+}$is a bounded continuous functional satisfying

$$
0 \leq \Phi[u] \leq K\|u\|_{L^{2}(\Omega)}^{2},
$$


where $K>0$ are constants independent of $u, \mathbb{R}^{+}=[0,+\infty)$,

$L_{+}^{2}(\Omega)=\left\{u \in L^{2}(\Omega) \mid u \geq 0\right.$, a.e. in $\left.\Omega\right\}$

(A2) $m(x, t) \in C_{T}\left(\bar{Q}_{T}\right)$ and satisfies that $\left\{x \in \Omega: \frac{1}{T} \int_{0}^{T} m(x, t)>0\right\} \neq \emptyset$, where $C_{T}\left(\bar{Q}_{T}\right)$ denotes the set of functions which are continuous in $\bar{\Omega} \times \mathbb{R}$ and of $T$-periodic with respect to $t$.

From (A2), we can see that there exist $x_{0} \in \Omega, \delta>0, m_{0}>0$ such that

$$
\frac{1}{T} \int_{0}^{T} m(x, t) d t \geq m_{0} \quad \text { for all } x \in B\left(x_{0}, \delta\right)
$$

Since equation (1.1) is degenerate at points where $u=0$, problem (1.1)-(1.3) has no classical solutions in general, so we focus on the discussion of weak solutions in the sense of the following.

Definition 1 A function $u$ is said to be a weak solution of problem (1.1)-(1.3) if $u \in$ $L^{p}\left(0, T ; W^{1, p}(\Omega)\right) \cap C_{T}\left(\bar{Q}_{T}\right)$ and satisfies

$$
\iint_{Q_{T}}\left(-u \frac{\partial \varphi}{\partial t}+|\nabla u|^{p-2} \nabla u \nabla \varphi-(m-\Phi[u]) u \varphi\right) d x d t=0
$$

for any $\varphi \in C^{1}\left(\bar{Q}_{T}\right)$ with $\varphi(x, 0)=\varphi(x, T)$.

Due to the degeneracy of equation (1.1), we consider the following regularized problem:

$$
\begin{aligned}
& \frac{\partial u_{\varepsilon}}{\partial t}-\operatorname{div}\left(\left(\left|\nabla u_{\varepsilon}\right|^{2}+\varepsilon\right)^{\frac{p-2}{2}} \nabla u_{\varepsilon}\right)+\varepsilon u_{\varepsilon}=\left(m-\Phi\left[u_{\varepsilon}\right]\right) u_{\varepsilon}^{+}, \quad(x, t) \in Q_{T}, \\
& \frac{\partial u_{\varepsilon}}{\partial n}=0, \quad(x, t) \in \partial \Omega \times(0, T), \\
& u_{\varepsilon}(x, 0)=u_{\varepsilon}(x, T), \quad x \in \Omega,
\end{aligned}
$$

where $s^{+}=\max \{0, s\}$ and $\varepsilon$ is a sufficiently small positive constant. The desired solution will be obtained as the limit point of the solutions of problem (2.2)-(2.4). In the following, we introduce a map by the following problem:

$$
\begin{aligned}
& \frac{\partial u_{\varepsilon}}{\partial t}-\operatorname{div}\left(\left(\left|\nabla u_{\varepsilon}\right|^{2}+\varepsilon\right)^{\frac{p-2}{2}} \nabla u_{\varepsilon}\right)+\varepsilon u_{\varepsilon}=f, \quad(x, t) \in Q_{T}, \\
& \frac{\partial u_{\varepsilon}}{\partial n}=0, \quad(x, t) \in \partial \Omega \times(0, T), \\
& u_{\varepsilon}(x, 0)=u_{\varepsilon}(x, T), \quad x \in \Omega .
\end{aligned}
$$

Then we can define a map $u_{\varepsilon}=G f$ with $G: C_{T}\left(\bar{Q}_{T}\right) \rightarrow C_{T}\left(\bar{Q}_{T}\right)$. Applying classical estimates (see [15]), we can know that $\left\|u_{\varepsilon}\right\|_{L^{\infty}\left(Q_{T}\right)}$ is bounded by $\|f\|_{L^{\infty}\left(Q_{T}\right)}$ and $u_{\varepsilon}$ is Hölder continuous in $Q_{T}$. Then by the Arzela-Ascoli theorem, the map $G$ is compact. So, the map is a compact continuous map. Let $f(u)=\left(m-\Phi\left[u_{\varepsilon}\right]\right) u_{\varepsilon}^{+}$, where $u_{\varepsilon}^{+}=\max \left\{u_{\varepsilon}, 0\right\}$, we can see that the nonnegative solution of problem (2.2)-(2.4) is also a nonnegative solution solving $u_{\varepsilon}=G\left(\left(m-\Phi\left[u_{\varepsilon}\right]\right) u_{\varepsilon}^{+}\right)$. So, we will study the existence of nonnegative fixed points of the map $u_{\varepsilon}=G\left(\left(m-\Phi\left[u_{\varepsilon}\right]\right) u_{\varepsilon}^{+}\right)$instead of the nonnegative solutions of problem (2.2)-(2.4). 


\section{Proof of the main results}

First, by the same method as in [14], we can obtain the nonnegativity of the solution of problem (2.2)-(2.4).

Lemma 1 If a nontrivial function $u_{\varepsilon} \in C_{T}\left(\bar{Q}_{T}\right)$ solves $u_{\varepsilon}=G\left(\left(m-\Phi\left[u_{\varepsilon}\right]\right) u_{\varepsilon}^{+}\right)$, then

$$
u_{\varepsilon}(x, t)>0 \quad \text { for all }(x, t) \in \bar{Q}_{T} .
$$

In the following, by the Moser iterative technique, we will show the a priori estimate for the upper bound of nonnegative periodic solutions of problem (2.5)-(2.7). Here and below we denote by $\|\cdot\|_{p}(1 \leq p \leq \infty)$ the $L^{p}(\Omega)$ norm.

Lemma 2 Let $\lambda \in[0,1], u_{\varepsilon}$ be a nonnegative periodic solution solving $u_{\varepsilon}=G(\lambda(m-$ $\left.\left.\Phi\left[u_{\varepsilon}\right]\right) u_{\varepsilon}^{+}\right)$, then there exists a constant $R>0$ independent of $\lambda, \varepsilon$ such that

$$
\left\|u_{\varepsilon}(t)\right\|_{\infty}<R
$$

where $u_{\varepsilon}(t)=u_{\varepsilon}(\cdot, t)$.

Proof Multiplying Eq. (2.5) by $u_{\varepsilon}^{m+1}(m \geq 0)$ and integrating over $\Omega$, we have

$$
\begin{aligned}
& \frac{1}{m+2} \frac{d}{d t}\left\|u_{\varepsilon}(t)\right\|_{m+2}^{m+2}+\frac{(m+1) p^{p}}{(m+p)^{p}}\left\|\nabla\left(u_{\varepsilon}^{\frac{m}{p}+1}(t)\right)\right\|_{p}^{p} \\
& \leq\|m(x, t)\|_{L^{\infty}(\Omega \times(0, T))}\left\|u_{\varepsilon}(t)\right\|_{m+2}^{m+2},
\end{aligned}
$$

and hence

$$
\begin{aligned}
& \frac{d}{d t}\left\|u_{\varepsilon}(t)\right\|_{m+2}^{m+2}+\frac{C_{1}}{(m+1)^{p-2}}\left\|\nabla\left(\left|u_{\varepsilon}(t)\right|^{\frac{m}{p}} u_{\varepsilon}(t)\right)\right\|_{p}^{p} \\
& \quad \leq C_{2}(m+1)\left\|u_{\varepsilon}(t)\right\|_{m+2}^{m+2}
\end{aligned}
$$

where $C_{i}(i=1,2)$ are positive constants independent of $u_{\varepsilon}$ and $m$.

Assume that $\left\|u_{\varepsilon}(t)\right\|_{\infty} \neq 0$ and set

$$
u_{k}(t)=\left|u_{\varepsilon}(t)\right|^{\frac{m_{k}}{p}} u_{\varepsilon}(t), \quad \alpha_{k}=\frac{p\left(m_{k}+2\right)}{m_{k}+p}, \quad m_{k}=\frac{p^{k}-p}{p-1} \quad(k=1,2, \ldots),
$$

then $\alpha_{k}<p, m_{k}=p^{k-1}+m_{k-1}$. For convenience, we denote by $C$ a positive constant independent of $k$ and $m$, which may take different values. From (3.2) we obtain

$$
\frac{d}{d t}\left\|u_{k}(t)\right\|_{\alpha_{k}}^{\alpha_{k}}+\frac{C}{(m+1)^{p-2}}\left\|\nabla u_{k}(t)\right\|_{p}^{p} \leq C(m+1)\left\|u_{k}(t)\right\|_{\alpha_{k}}^{\alpha_{k}} .
$$

By using the Gagliardo-Nirenberg inequality, we have

$$
\left\|u_{k}(t)\right\|_{\alpha_{k}} \leq C\left\|\nabla u_{k}(t)\right\|_{p}^{\theta_{k}}\left\|u_{k}(t)\right\|_{1}^{1-\theta_{k}},
$$


with

$$
\theta_{k}=\frac{(p-1) m_{k}+p}{m_{k}+2} \cdot \frac{N}{(p-1) N+p} \in(0,1) .
$$

By inequalities (3.3), (3.4) and the fact that $\left\|u_{k}(t)\right\|_{1}=\left\|u_{k-1}(t)\right\|_{\alpha_{k-1}}^{\alpha_{k-1}}$, we obtain the following differential inequality:

$$
\begin{aligned}
\frac{d}{d t}\left\|u_{k}(t)\right\|_{\alpha_{k}}^{\alpha_{k}} & \leq-\frac{C}{\left(m_{k}+1\right)^{p-2}}\left\|u_{k}(t)\right\|_{\alpha_{k}}^{\frac{p}{\theta_{k}}}\left\|u_{k}(t)\right\|_{1}^{\frac{p\left(\theta_{k}-1\right)}{\theta_{k}}}+C\left(m_{k}+1\right)\left\|u_{k}(t)\right\|_{\alpha_{k}}^{\alpha_{k}} \\
& \leq-C\left\|u_{k}(t)\right\|_{\alpha_{k}}^{\frac{p}{\theta_{k}}}\left\|u_{k-1}(t)\right\|_{\alpha_{k-1}}^{\frac{\theta_{k-1}}{\theta_{k}} \alpha_{k-1} p}+C\left(m_{k}+1\right)\left\|u_{k}(t)\right\|_{\alpha_{k}}^{\alpha_{k}} .
\end{aligned}
$$

Let

$$
\lambda_{k}=\max \left\{1, \sup _{t}\left\|u_{k}(t)\right\|_{\alpha_{k}}\right\}
$$

we have

$$
\begin{aligned}
\frac{d}{d t}\left\|u_{k}(t)\right\|_{\alpha_{k}}^{\alpha_{k}} \leq & \left(m_{k}+1\right)^{-(p-2)}\left\|u_{k}(t)\right\|_{\alpha_{k}}^{\frac{\alpha_{k}\left(m_{k}+1\right)}{m_{k}+2}}\left\{-C\left\|u_{k}(t)\right\|_{\alpha_{k}}^{\frac{p}{\theta_{k}}-\frac{\alpha_{k}\left(m_{k}+1\right)}{m_{k}+2}} \lambda_{k-1}^{\frac{\theta_{k}-1}{\theta_{k}} \alpha_{k-1} p}\right. \\
& \left.+C\left(m_{k}+1\right)^{p-1}\left\|u_{k}(t)\right\|_{\alpha_{k}}^{\frac{\alpha_{k}}{m_{k}+2}}\right\} .
\end{aligned}
$$

For Young's inequality

$$
a b \leq \varepsilon a^{p^{\prime}}+\varepsilon^{-\frac{q^{\prime}}{p^{\prime}}} b^{q^{\prime}}
$$

where $p^{\prime}>1, q^{\prime}>1, a>0, b>0, \varepsilon>0$ and $\frac{1}{p^{\prime}}+\frac{1}{q^{\prime}}=1$, we set

$$
\begin{aligned}
& a=\left\|u_{k}(t)\right\|_{\alpha_{k}}^{\frac{\alpha_{k}}{m_{k}+2}}, \quad b=\left(m_{k}+1\right)^{p-1}, \quad \varepsilon=\frac{1}{2} \lambda_{k-1}^{\frac{\theta_{k}-1}{\theta_{k}} \alpha_{k-1} p}, \\
& p^{\prime}=l_{k}=\frac{p(m+2)}{\theta_{k} \alpha_{k}}-m_{k}-1=\frac{\left.\left(m_{k}+2\right)\left(m_{k}+p\right)(p-1) N+p\right)}{N\left((p-1) m_{k}+p\right)}-m_{k}-1,
\end{aligned}
$$

then we have

$$
\begin{aligned}
\left(m_{k}+1\right)^{p-1}\left\|u_{k}(t)\right\|_{\alpha_{k}}^{\frac{\alpha_{k}}{m_{k}+2}} \leq & \frac{1}{2}\left\|u_{k}(t)\right\|_{\alpha_{k}}^{\frac{p}{\theta_{k}}-\frac{\alpha_{k}\left(m_{k}+1\right)}{m_{k}+2}} \lambda_{k-1}^{\frac{\theta_{k}-1}{\theta_{k}} \alpha_{k-1} p} \\
& +C\left(m_{k}+1\right)^{p-1} l_{k_{k}^{-1}}^{\frac{l_{k}}{l_{k}}} \lambda_{k-1}^{\frac{1-\theta_{k}}{\theta_{k}} \alpha_{k-1} p \frac{1}{l_{k}-1}} .
\end{aligned}
$$

Here we have used the fact that $p^{\prime}=l_{k}>r>1$ for some $r$ independent of $k$. In fact, it is easy to verify that

$$
\lim _{k \rightarrow \infty} l_{k}=+\infty
$$

Denoting

$$
a_{k}=\frac{(p-1) l_{k}}{l_{k}-1}, \quad b_{k}=\frac{1-\theta_{k}}{\theta_{k}} \frac{p \alpha_{k-1}}{l_{k}-1}
$$


and combining (3.6), (3.5), we obtain

$$
\begin{aligned}
\frac{d}{d t}\left\|u_{k}(t)\right\|_{\alpha_{k}}^{\alpha_{k}} \leq & \left(m_{k}+1\right)^{-(p-2)}\left\|u_{k}(t)\right\|_{\alpha_{k}}^{\frac{\alpha_{k}\left(m_{k}+1\right)}{m_{k}+2}}\left\{\frac{-C}{2}\left\|u_{k}(t)\right\|_{\alpha_{k}}^{\frac{p}{\theta_{k}}-\frac{\alpha_{k}\left(m_{k}+1\right)}{m_{k}+2}} \lambda_{k-1}^{\frac{\theta_{k}-1}{\theta_{k}} \alpha_{k-1} p}\right. \\
& \left.+C\left(m_{k}+1\right)^{a_{k}} \lambda_{k-1}^{b_{k}}\right\}
\end{aligned}
$$

that is,

$$
\begin{aligned}
\left(m_{k}+1\right)^{p-2}\left(m_{k}+1\right) \frac{d}{d t}\left\|u_{k}(t)\right\|_{\alpha_{k}}^{\frac{\alpha_{k}}{m_{k}+2}} \leq & \frac{-C}{2}\left\|u_{k}(t)\right\|_{\alpha_{k}}^{\frac{p}{\theta_{k}}-\frac{\alpha_{k}\left(m_{k}+1\right)}{m_{k}+2}} \lambda_{k-1}^{\frac{\theta_{k}-1}{\theta_{k}} \alpha_{k-1} p} \\
& +C\left(m_{k}+1\right)^{a_{k}} \lambda_{k-1}^{b_{k}} .
\end{aligned}
$$

From the periodicity of $u_{k}(t)$, we know that there exists $t_{0}$ at which $\left\|u_{k}(t)\right\|_{\alpha_{k}}$ reaches its maximum and thus the left-hand side of (3.8) vanishes. Then we obtain

$$
\left\|u_{k}(t)\right\|_{\alpha_{k}} \leq\left\{C\left[\left(m_{k}+1\right)^{p-1}+\left(m_{k}+1\right)^{a_{k}} \lambda_{k-1}^{b_{k}} \lambda_{k-1}^{\frac{1-\theta_{k}}{\theta_{k}} \alpha_{k-1} p}\right]\right\}^{\frac{1}{\alpha_{k}}}
$$

where

$$
\alpha_{k}=\frac{p}{\theta_{k}}-\frac{\alpha_{k}\left(m_{k}+1\right)}{m_{k}+2}=\frac{l_{k} \alpha_{k}}{m_{k}+2} .
$$

Therefore we conclude that

$$
\left\|u_{k}(t)\right\|_{\alpha_{k}} \leq\left\{C\left(m_{k}+1\right)^{a_{k}} \lambda_{k-1}^{b_{k}+\frac{1-\theta_{k}}{\theta_{k}} \alpha_{k-1} p}\right\}^{\frac{1}{C_{k}}}=\left\{C\left(m_{k}+1\right)^{a_{k}}\right\}^{\frac{m_{k}+2}{l_{k} \alpha_{k}}} \lambda^{\frac{\left(1-\theta_{k}\right)\left(m_{k+2}\right) \alpha_{k-1} p}{\left(l_{k}-1\right) \theta_{k} \alpha_{k}}} .
$$

Since $\frac{m_{k}+2}{\left(l_{k}-1\right) \theta_{k}}=\frac{\alpha_{k}}{p-\theta_{k} \alpha_{k}}$ and $\frac{m_{k}+2}{l_{k} \alpha_{k}}$ and $a_{k}$ are bounded, we have

$$
\left\|u_{k}(t)\right\|_{\alpha_{k}} \leq C p^{k a^{\prime}} \lambda_{k-1}^{\frac{\left(1-\theta_{k}\right) \alpha_{k-1} p}{p-\theta_{k} \alpha_{k}}}
$$

where $a^{\prime}$ is a positive constant independent of $k$. As $\alpha_{k}=\frac{p\left(m_{k}+2\right)}{m_{k}+p}<p$ implies that $\frac{\left(1-\theta_{k}\right) \alpha_{k-1} p}{p-\theta_{k} \alpha_{k}} \leq \frac{\left(1-\theta_{k}\right) \alpha_{k-1} p}{p-p \theta_{k}} \leq p$ and $\lambda_{k-1} \geq 1$, we get

$$
\left\|u_{k}(t)\right\|_{\alpha_{k}} \leq C A^{k} \lambda_{k-1}^{p}
$$

or

$$
\ln \left\|u_{k}(t)\right\|_{\alpha_{k}} \leq \ln \lambda_{k} \leq \ln C+k \ln A+p \ln \lambda_{k-1}
$$

where $A=p^{a^{\prime}}>1$. Thus

$$
\begin{aligned}
\ln \left\|u_{k}(t)\right\|_{\alpha_{k}} & \leq \ln C \sum_{i=0}^{k-2} p^{i}+p^{k-1} \ln l_{1}+\ln A\left(\sum_{j=0}^{k-2}(k-j) p^{j}\right) \\
& \leq \frac{p^{k-1}-1}{p-1} \ln C+p^{k-1} \ln l_{1}+f(k) \ln A,
\end{aligned}
$$


or

$$
\left\|u_{\varepsilon}(t)\right\|_{m_{k}+2} \leq\left\{C^{\frac{p^{k-1}-1}{p-1}} l_{1}^{p^{k-1}} A f(k)\right\}^{\frac{p}{m_{k}+p}}
$$

where

$$
f(k)=\frac{k-(k+1) p-p^{k-1}+2 p^{k}}{(p-1)^{2}} .
$$

Letting $k \rightarrow \infty$, we obtain

$$
\left\|u_{\varepsilon}(t)\right\|_{\infty} \leq C l_{1}^{p-1} \leq C\left(\max \left\{1, \sup _{t}\left\|u_{\varepsilon}(t)\right\|_{2}\right\}\right)^{p-1}
$$

On the other hand, it follows from (3.2) with $m=0$ that

$$
\frac{d}{d t}\left\|u_{\varepsilon}(t)\right\|_{2}^{2}+C_{1}\left\|\nabla u_{\varepsilon}(t)\right\|_{p}^{p} \leq C_{2}\left\|u_{\varepsilon}(t)\right\|_{2}^{2}
$$

By Hölder's inequality and Sobolev's theorem, we have

$$
\left\|u_{\varepsilon}(t)\right\|_{2} \leq|\Omega|^{\frac{1}{2}-\frac{1}{p}}\left\|u_{\varepsilon}(t)\right\|_{p} \leq C|\Omega|^{\frac{1}{2}-\frac{1}{p}}\left\|\nabla u_{\varepsilon}(t)\right\|_{p} .
$$

Combined with (3.10), it yields

$$
\frac{d}{d t}\left\|u_{\varepsilon}(t)\right\|_{2}^{2}+C_{1}\left\|\nabla u_{\varepsilon}(t)\right\|_{2}^{p} \leq C_{2}\left\|u_{\varepsilon}(t)\right\|_{2}^{2}
$$

By Young's inequality, it follows that

$$
\frac{d}{d t}\left\|u_{\varepsilon}(t)\right\|_{2}^{2}+C_{1}\left\|\nabla u_{\varepsilon}(t)\right\|_{2}^{p} \leq C_{2}
$$

where $C_{i}(i=1,2)$ are constants independent of $u$. Taking the periodicity of $u$ into account, we infer from (3.12) that

$$
\left\|u_{\varepsilon}(t)\right\|_{2} \leq C
$$

which together with (3.9) implies (3.1). The proof is completed.

Corollary 1 There exists a positive constant $R$ independent of $\varepsilon$ such that

$$
\operatorname{deg}\left(I-G\left(\left(m-\Phi\left[u_{\varepsilon}\right]\right) u_{\varepsilon}^{+}\right), B_{R}, 0\right)=1,
$$

where $B_{R}$ is a ball centered at the origin with radius $R$ in $L^{\infty}\left(Q_{T}\right)$.

Proof It follows from Lemma 2 that there exists a positive constant $R$ independent of $\varepsilon$ such that

$$
u_{\varepsilon} \neq G\left(\lambda\left(m-\Phi\left[u_{\varepsilon}\right]\right) u_{\varepsilon}^{+}\right), \quad \forall u_{\varepsilon} \in \partial B_{R}, \lambda \in[0,1]
$$


Hence the degree is well defined on $B_{R}$. From the homotopy invariance of the LeraySchauder degree, we can see that

$$
\operatorname{deg}\left(I-G\left(\left(m-\Phi\left[u_{\varepsilon}\right]\right) u_{\varepsilon}^{+}\right), B_{R}, 0\right)=\operatorname{deg}\left(I-G(0), B_{R}, 0\right) .
$$

From the existence and uniqueness of the solution of $u_{\varepsilon}=G(0)$, we have $\operatorname{deg}(I-$ $\left.G(0), B_{R}, 0\right)=1$. That is, $\operatorname{deg}\left(I-G\left(\left(m-\Phi\left[u_{\varepsilon}\right]\right) u_{\varepsilon}^{+}\right), B_{R}, 0\right)=1$. The proof is completed.

Lemma 3 There exist constants $r_{0}>0$ and $\varepsilon_{0}>0$ such that for any $r<r_{0}, \varepsilon<\varepsilon_{0}, G((m-$ $\left.\Phi\left[u_{\varepsilon}\right]\right) u_{\varepsilon}^{+}$) admits no nontrivial solution $u_{\varepsilon}$ satisfying

$$
0<\left\|u_{\varepsilon}\right\|_{L^{\infty}\left(Q_{T}\right)} \leq r
$$

where $r$ is a positive constant independent of $\varepsilon$.

Proof By contradiction, let $u_{\varepsilon}$ be a nontrivial solution of $u_{\varepsilon}=G\left(\left(m-\Phi\left[u_{\varepsilon}\right]\right) u_{\varepsilon}^{+}\right)$satisfying $0<\left\|u_{\varepsilon}\right\|_{L^{\infty}\left(Q_{T}\right)} \leq r$. For any given $\phi(x) \in C_{0}^{\infty}(\Omega)$, multiplying (2.5) by $\frac{\phi^{2}}{u_{\varepsilon}}$ and integrating over $Q_{T}^{*}=B_{\delta}\left(x_{0}\right) \times(0, T)$, we obtain

$$
\begin{aligned}
& \iint_{Q_{T}^{*}} \frac{\phi^{2}}{u_{\varepsilon}} \frac{\partial u_{\varepsilon}}{\partial t} d t d x+\iint_{Q_{T}^{*}}\left(\left(\left|\nabla u_{\varepsilon}\right|^{2}+\varepsilon\right)^{\frac{p-2}{2}} \nabla u_{\varepsilon} \nabla\left(\frac{\phi^{2}}{u_{\varepsilon}}\right)\right) d t d x \\
& \quad=\iint_{Q_{T}^{*}} \phi^{2}\left(m-\varepsilon-\Phi\left[u_{\varepsilon}\right]\right) d t d x .
\end{aligned}
$$

By the periodicity of $u_{\varepsilon}$, the first term on the left-hand side in (3.14) is zero. As in the proof of Lemma 2.2 of [14], the second term on the left-hand side in (3.14) can be rewritten as

$$
\begin{aligned}
& \iint_{Q_{T}^{*}}\left(\left(\left|\nabla u_{\varepsilon}\right|^{2}+\varepsilon\right)^{\frac{p-2}{2}} \nabla u_{\varepsilon} \nabla\left(\frac{\phi^{2}}{u_{\varepsilon}}\right)\right) d t d x \\
& =\iint_{Q_{T}^{*}}\left(\left(\left|\nabla u_{\varepsilon}\right|^{2}+\varepsilon\right)^{\frac{p-2}{2}}|\nabla \phi|^{2}\right) d t d x \\
& \quad-\iint_{Q_{T}^{*}}\left(\left(\left|\nabla u_{\varepsilon}\right|^{2}+\varepsilon\right)^{\frac{p-2}{2}} u_{\varepsilon}^{2}\left|\nabla\left(\frac{\phi}{u_{\varepsilon}}\right)\right|^{2}\right) d t d x,
\end{aligned}
$$

and thus

$$
\begin{gathered}
\iint_{Q_{T}^{*}}\left(\left(\left|\nabla u_{\varepsilon}\right|^{2}+\varepsilon\right)^{\frac{p-2}{2}} \nabla u_{\varepsilon} \nabla\left(\frac{\phi^{2}}{u_{\varepsilon}}\right)\right) d t d x \\
\quad \leq \iint_{Q_{T}^{*}}\left(\left(\left|\nabla u_{\varepsilon}\right|^{2}+\varepsilon\right)^{\frac{p-2}{2}}|\nabla \phi|^{2}\right) d t d x .
\end{gathered}
$$

Combining (3.15) with (3.14), we obtain

$$
\iint_{Q_{T}^{*}} \phi^{2}\left(m-\varepsilon-\Phi\left[u_{\varepsilon}\right]\right) d t d x \leq \iint_{Q_{T}^{*}}\left(\left(\left|\nabla u_{\varepsilon}\right|^{2}+\varepsilon\right)^{\frac{p-2}{2}}|\nabla \phi|^{2}\right) d t d x .
$$


Let $\mu_{1}$ be the first eigenvalue of the $p$-Laplacian equation on $\Omega$ with zero boundary conditions and $\phi_{1}(x)$ be the corresponding eigenfunction. We have

$$
\int_{\Omega}\left|\nabla \phi_{1}\right|^{p} d x=\mu_{1} \int_{\Omega}\left|\phi_{1}\right|^{p} d x
$$

And also we know that $\phi_{1}$ can be strictly positive in the subfield $B_{\delta}\left(x_{0}\right) \subset \Omega$. Taking $\phi=\phi_{1}$, we have

$$
\begin{aligned}
& \iint_{Q_{T}^{*}}\left(\phi^{2}\left(m-\varepsilon-\Phi\left[u_{\varepsilon}\right]\right)\right) d t d x \\
& \quad \leq \iint_{Q_{T}^{*}}\left(\left(\left|\nabla u_{\varepsilon}\right|^{2}+\varepsilon\right)^{\frac{p-2}{2}}\left|\nabla \phi_{1}\right|^{2}\right) d x d t \\
& \quad \leq\left(\iint_{Q_{T}^{*}}\left(\left|\nabla u_{\varepsilon}\right|^{2}+\varepsilon\right)^{\frac{p}{2}} d x d t\right)^{\frac{p-2}{p}}\left(\iint_{Q_{T}^{*}}\left|\nabla \phi_{1}\right|^{p} d x d t\right)^{\frac{2}{p}} \\
& \quad \leq\left(\iint_{Q_{T}^{*}} 2^{\frac{p}{2}}\left(\left|\nabla u_{\varepsilon}\right|^{p}+\varepsilon^{\frac{p}{2}}\right) d x d t\right)^{\frac{p-2}{p}}\left(T \mu_{1} \int_{B_{\delta}\left(x_{0}\right)}\left|\phi_{1}\right|^{p} d x\right)^{\frac{2}{p}} .
\end{aligned}
$$

Multiplying (2.5) by $u_{\varepsilon}$ and integrating over $Q_{T}^{*}$, from the assumption $0<\left\|u_{\varepsilon}\right\|_{L^{\infty}\left(Q_{T}\right)} \leq r$, we have

$$
\iint_{Q_{T}^{*}}\left|\nabla u_{\varepsilon}\right|^{p} d x d t \leq \iint_{Q_{T}^{*}} u_{\varepsilon}^{2}\left(m-\varepsilon-\Phi\left[u_{\varepsilon}\right]\right) d x d t \leq M \operatorname{Tr}^{2}|\Omega|
$$

where $M=\max _{(x, t) \in Q_{T}^{*}} m(x, t)$ and $|\Omega|$ denotes the Lebesgue measure of the domain $\Omega$. Combining (3.18) with (3.17), we obtain

$$
\begin{aligned}
& \iint_{Q_{T}^{*}}\left(\phi^{2}\left(m-\varepsilon-\Phi\left[u_{\varepsilon}\right]\right)\right) d t d x \\
& \quad \leq 2^{\frac{p-2}{p}} T \mu_{1}^{\frac{2}{p}}|\Omega|^{\frac{p-2}{p}}\left(M r^{2}+\varepsilon^{\frac{2}{p}}\right)\left(\int_{B_{\delta}\left(x_{0}\right)}\left|\phi_{1}\right|^{p} d x\right)^{\frac{2}{p}} .
\end{aligned}
$$

In addition, the assumptions (A1), (A2) give

$$
\begin{aligned}
\iint_{Q_{T}^{*}} \phi_{1}^{2}\left(m-\varepsilon-\Phi\left[u_{\varepsilon}\right]\right) d x d t & \geq \iint_{Q_{T}^{*}} \phi_{1}^{2}\left(m-\varepsilon-K\|u\|_{L^{2}}^{2}\right) d x d t \\
& \geq \int_{B_{\delta}\left(x_{0}\right)} \phi_{1}^{2} \int_{0}^{T}\left(m-\varepsilon-K\|u\|_{L^{2}}^{2}\right) d t d x \\
& \geq T\left(m_{0}-\varepsilon-K r^{2}|\Omega|\right) \int_{B_{\delta}\left(x_{0}\right)} \phi_{1}^{2} d x .
\end{aligned}
$$

The above two inequalities imply that

$$
m_{0}-\varepsilon-K r^{2}|\Omega| \leq 2^{\frac{p-2}{p}} \mu_{1}^{\frac{2}{p}}|\Omega|^{\frac{p-2}{p}}\left(M r^{2}+\varepsilon^{\frac{2}{p}}\right) \frac{\left(\int_{B_{\delta}\left(x_{0}\right)}\left|\phi_{1}\right|^{p} d x\right)^{\frac{2}{p}}}{\int_{B_{\delta}\left(x_{0}\right)} \phi_{1}^{2} d x} .
$$

Obviously, we can choose suitably small $\varepsilon_{0}>0$ and $r_{0}>0$ such that for any $\varepsilon \leq \varepsilon_{0}, r \leq r_{0}$, the inequality (3.21) does not hold. It is a contradiction. The proof is completed. 
Corollary 2 There exists a small positive constant $r$ which is independent of $\varepsilon$ and satisfies $r<R$ such that

$$
\operatorname{deg}\left(I-G\left(\left(m-\Phi\left[u_{\varepsilon}\right]\right) u_{\varepsilon}^{+}\right), B_{r}, 0\right)=0,
$$

where $B_{r}$ is a ball centered at the origin with radius $r$ in $L^{\infty}\left(Q_{T}\right)$.

Proof Similar to Lemma 3, we can see that there exists a positive constant $0<r<R$ independent of $\varepsilon$ such that

$$
u_{\varepsilon} \neq G\left(\lambda\left(m-\Phi\left[u_{\varepsilon}\right]\right) u_{\varepsilon}^{+}+1-\lambda\right), \quad \forall u_{\varepsilon} \in \partial B_{r}, \lambda \in[0,1] .
$$

Hence the degree is well defined on $B_{r}$. From the homotopy invariance of the LeraySchauder degree, we can see that

$$
\operatorname{deg}\left(I-G\left(\left(m-\Phi\left[u_{\varepsilon}\right]\right) u_{\varepsilon}^{+}\right), B_{r}, 0\right)=\operatorname{deg}\left(I-G(1), B_{r}, 0\right) .
$$

Lemma 3 shows that $u_{\varepsilon}=G(1)$ admits no nontrivial solution in $B_{r}$ and it is also easy to see that $u_{\varepsilon}=0$ is not a solution of $u_{\varepsilon}=G(1)$. So, we have $\operatorname{deg}(I-G(1), B r, 0)=0$, that is,

$$
\operatorname{deg}\left(I-G\left(\left(m-\Phi\left[u_{\varepsilon}\right]\right) u_{\varepsilon}^{+}\right), B_{r}, 0\right)=0 .
$$

The proof is completed.

Theorem 1 Ifassumptions (A1) and (A2) hold, then problem (1.1)-(1.3) admits a nontrivial nonnegative periodic solution $u$.

Proof Using Corollary 1 and Corollary 2, we know that

$$
\operatorname{deg}(I-G(f(\cdot)), \Sigma, 0)=1,
$$

where $\Sigma=B_{R} \backslash B_{r}, B_{\xi}$ is a ball centered at the origin with radius $\xi$ in $L^{\infty}\left(Q_{T}\right), R$ and $r$ are positive constants and $R>r$. By the theory of the Leray-Schauder degree and Lemma 1, we can conclude that problem (2.2)-(2.4) admits a nontrivial nonnegative periodic solution $u_{\varepsilon}$. By Lemma 3 and a similar method to that in [14], we can obtain

$$
\left\|\nabla u_{\varepsilon}\right\|_{L^{p}\left(Q_{T}\right)} \leq C, \quad\left\|\frac{\partial u_{\varepsilon}}{\partial t}\right\|_{L^{2}\left(Q_{T}\right)} \leq C .
$$

Combining with the regularity results [16] a similar argument to that in [17], we can prove that the limit function of $u_{\varepsilon}$ is a nonnegative nontrivial periodic solution of problem (1.1)(1.3). 
Authors' contributions

$\mathrm{RH}, \mathrm{JS}$ and BW carried out the proof of the main part of this article, BW corrected the manuscript and participated in its design and coordination. All authors have read and approved the final manuscript.

\section{Author details}

${ }^{1}$ Department of Mathematics, Harbin Institute of Technology, Harbin, 150001, P.R. China. ${ }^{2}$ Department of Mathematics, College of Education, Tikrit University, Tikrit, Iraq.

\section{Acknowledgements}

This work is partially supported by the National Science Foundation of China (11271100, 11126222), the Fundamental Research Funds for the Central Universities (Grant No. HIT. NSRIF. 2011006), the Natural Sciences Foundation of Heilongjiang Province (QC2011C020) and also the 985 project of Harbin Institute of Technology.

Received: 6 March 2013 Accepted: 7 June 2013 Published: 23 July 2013

\section{References}

1. Allegretto, W, Nistri, P: Existence and optimal control for periodic parabolic equations with nonlocal term. IMA J. Math. Control Inf. 16(1), 43-58 (1999)

2. Huang, R, Wang, Y, Ke, Y: Existence of the non-trivial nonnegative periodic solutions for a class of degenerate parabolic equations with nonlocal terms. Discrete Contin. Dyn. Syst. 5(4), 1005-1014 (2005)

3. Ke, Y, Huang, R, Sun, J: Periodic solutions for a degenerate parabolic equation. Appl. Math. Lett. 22, $910-915$ (2009)

4. Lieberman, GM: Time-periodic solutions of quasilinear parabolic differential equations I. Dirichlet boundary conditions. J. Math. Anal. Appl. 264(2), 617-638 (2001)

5. Li, J, Sun, J, Wu, B: Periodic doubly degenerate parabolic equation with nonlocal terms. Comput. Math. Appl. 60 490-500 (2010)

6. Liu, Z: Periodic solutions for double degenerate quasilinear parabolic equations. Nonlinear Anal. TMA 51(7), 1245-1257 (2002)

7. Zhou, J, Mu, C: Time periodic solutions of porous medium equation. Math. Methods Appl. Sci. (2010) doi:10.1002/mma.1307

8. Wang, YF, Yin, JX: Periodic solutions for a class of degenerate parabolic equations with Neumann boundary conditions. Nonlinear Anal., Real World Appl. 12, 2069-2076 (2011)

9. Sun, JB, Yin, JX, Wang, YF: Asymptotic bounds of solutions for a periodic doubly degenerate parabolic equation. Nonlinear Anal. 74(6), 2415-2424 (2011)

10. Pang, PYH, Wang, YF, Yin, JX: Periodic solutions for a class of reaction-diffusion equations with $p$-Laplacian. Nonlinear Anal., Real World Appl. 11(1), 323-331 (2010)

11. Wang, YF, Wu, ZQ, Yin, JX: Periodic solutions of evolution $p$-Laplacian equations with weakly nonlinear sources. Int. J. Math. Game Theory Algebr. 10(1), 67-77 (2000)

12. Georgiev, S: Periodic solutions to the nonlinear parabolic equation. Int. J. Evol. Equ. 5(1), 53-66 (2010)

13. Klimov, VS: Periodic solutions of parabolic inclusions and the averaging method. Differ. Uravn. (Minsk) 46(12) $1722-1730(2010)$

14. Zhou, Q, Ke, YY, Wang, YF, Yin, JX: Periodic $p$-Laplacian with nonlocal terms. Nonlinear Anal. 66, 442-453 (2007)

15. Ladyzhenskaja, OA, Solonnikov, VA, Ural'ceva, NN: Linear and Quasilinear Equations of Parabolic Type. Transl. Math. Monogr., vol. 23. Am. Math. Soc., Providence (1968)

16. Dibenedetto, E: Degenerate Parabolic Equations. Springer, New York (1993)

17. Wu, ZQ, Zhao, JN, Yin, JX, Li, HL: Nonlinear Diffusion Equation. World Scientific, Singapore (2001)

doi:10.1186/1687-2770-2013-171

Cite this article as: Hameed et al.: Existence of periodic solutions of a $p$-Laplacian-Neumann problem. Boundary Value Problems 2013 2013:171.

\section{Submit your manuscript to a SpringerOpen ${ }^{\ominus}$ journal and benefit from:}

- Convenient online submission

- Rigorous peer review

- Immediate publication on acceptance

Open access: articles freely available online

- High visibility within the field

- Retaining the copyright to your article 